\title{
Synthesis and Characterization of tris-(4-Phenoxyphenyl)amine by Conventional Method
}

\author{
RAJESH KUMAR MALIK ${ }^{1 *}$, JTTENDER KUMAR NARWAL ${ }^{2}$ and \\ SURENDRA KUMAR and ANURADHA ${ }^{2}$ \\ ${ }^{1}$ Department of Chemistry, M.D University, Rohtak-124001, India \\ ${ }^{2}$ Department of Chemistry, JVMGRR College, Charkhi Dadri, Bhiwani, India \\ jitender.narwal@yahoo.com
}

Received 1 June 2016 / Accepted 26 June 2016

\begin{abstract}
Conjugated and non-conjugated compounds were synthesized. The compounds were synthesized by two step reaction i.e. (1) by direct bromination of the organic compound followed by N/O-alkyl/arylation and (2) by bromomethylation of the organic compound followed by N/Oalkyl/arylation.
\end{abstract}

Keywords: Synthesis, Organic electroluminescence, Conjugated, Non-conjugated compounds

\section{Introduction}

Organic electroluminescence (EL) is the electrically driven emission of light from noncrystalline organic materials, which was first observed and extensively studied in the $1960 \mathrm{~s}^{1,2}$. In 1987, a research group in Kodak introduced a double layer organic lightemitting diodes (OLEDs), which combined modern thin film deposition techniques with suitable materials and structure to give moderately low bias voltages and attractive luminance efficiency,4. Later in 1990 the Cambridge Group of Friend announced the first conducting polymer-based LED $^{5,6}$. Since after that, there have been tremendous research activities in this new field and enormous progress have been made in the improvements of color gamut, luminance efficiency and device reliability. The growing interest is largely motivated by the promise of the use of this technology toward full-color flat panel displays.

The amorphous nature of the TPA containing materials can be enhanced by the attachment of bulky substituents or the generation of more extended TPA oligomers via connection by para-linkages. The star shaped triphenylamine structures leads to a decreased crystallization tendency due to bulky, non polar geometry. Synthesized number of low oxidation potential star-shaped oligo triarylamine compounds with different cores (Figure 1). 
<smiles>c1ccc(N(c2ccccc2)c2ccccc2)cc1</smiles>

TPA<smiles>C1=CC(=[N+](c2ccccc2)c2ccccc2)C=CC1</smiles>

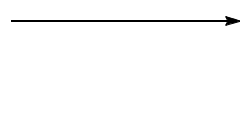

\section{Experimental}

Triphenylamine was purchased from Sigma Aldrich India, bromine, phenol, 18-crown-6ether, potassium carbonate were purchased from Spectrochem chemicals India Pvt. Ltd. The solvents were distilled off and dried by the conventional known methods before their use.

\section{Characterization}

${ }^{1} \mathrm{H}$ NMR and ${ }^{13} \mathrm{C}$ NMR were recorded on JEOL-400MHz NMR in the University Science Instrumentation Centre (USIC), Faculty of Science, University of Delhi. FT-IR was recorded on Perkin Elmer FT-IR spectrometer from the Department of Chemistry, University of Delhi. The UV-Vis absorption spectrum was recorded by Shimazu UV spectrophotometer and the florescence spectra was recorded by Cary Ecllipse florescence spectrophotometer from University Science Instrumentation Centre (USIC), Faculty of Science, University of Delhi.

\section{Synthesis ${ }^{7}$ of tris-(4-bromophenyl)amine}

Triphenylamine $(1.2 \mathrm{~g}, 5 \mathrm{mmol})$ was dissolved in $5 \mathrm{~mL}$ of chloroform and cooled down to $0{ }^{\circ} \mathrm{C}$. After half hour bromine $(0.5 \mathrm{~mL}$ dissolved in $3 \mathrm{~mL}$ chloroform, $20 \mathrm{mmol})$ was added to the reaction mixture and the resulting green solution was stirred for another one hour. The reaction mixture was poured in $20 \mathrm{~mL}(1: 1)$ ethanol:water mixture to precipitate out the product. The solid was filtered off under vacuum and dissolved in $4 \mathrm{~mL}$ hot chloroform. After addition of 15 $\mathrm{mL}$ hot ethanol the product was allowed to crystalline over night at $-18^{\circ} \mathrm{C}$. The synthesis of the organic compound was confirmed with melting point $142{ }^{\circ} \mathrm{C}$ and ${ }^{1} \mathrm{H}$ NMR shown in Figure 2.

\section{Synthesis of tris-(4-phenoxyphenyl)amine}

Tris-(4-bromophenyl)amine ( $1 \mathrm{~g}, 2 \mathrm{mmol})$, phenol (0.6 mL, $6 \mathrm{mmol})$, 18-crown-6-ether $(1.5$ g) and $\mathrm{K}_{2} \mathrm{CO}_{3}(1 \mathrm{~g})$ were dissolved in $10 \mathrm{~mL}$ acetone and allowed to reflux at $50{ }^{\circ} \mathrm{C}$ for $60 \mathrm{~h}$ (Scheme 1). The reaction progress was monitored by TLC in hexane : ethyl acetate (70:30\%). After this the reaction was quenched by adding $100 \mathrm{~mL}$ distilled water and organic compound was extracted using chloroform $(4 \times 50 \mathrm{~mL})$. The organic layer was dried over sodium sulfate and the solvent was removed under rotavapor. The compound was purified by column 
chromatography (Hexane: Ethyl acetate, 80:20) and pale yellow colored solid with melting point $129{ }^{\circ} \mathrm{C}$ was obtained. The compound was further confirmed by and ${ }^{1} \mathrm{H}$ NMR and ${ }^{13} \mathrm{C}$ NMR shown in Figure $3 \& 4$.

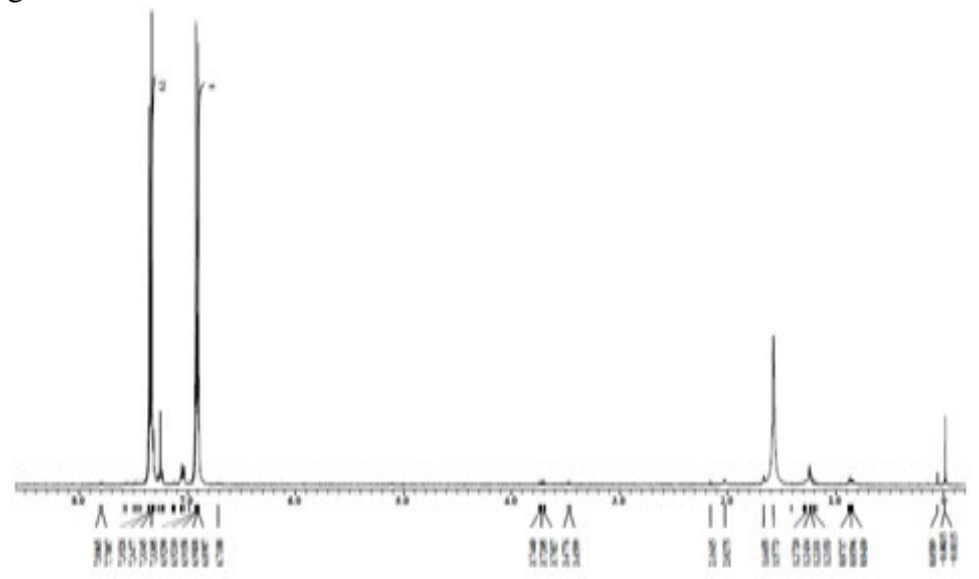

Figure 2. ${ }^{1} \mathrm{H}$ NMR for tris-(4-bromophenyl)amine

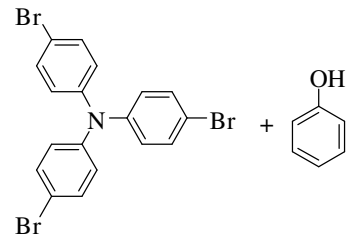

Tris-(4-bromophenyl)amine

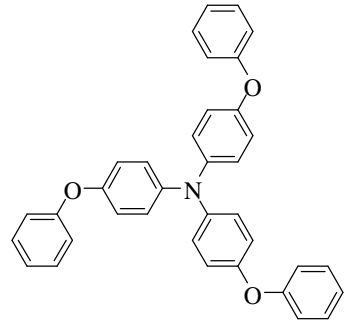

Tris-(4-phenoxyphenyl)amine

\section{Scheme 1}
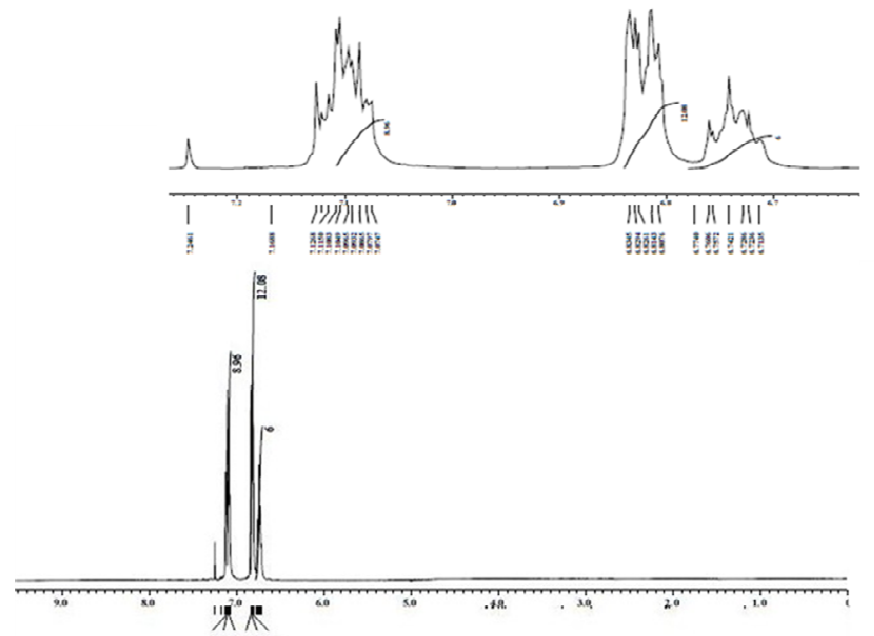

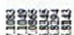

Figure 3. ${ }^{1} \mathrm{H}$ NMR for tris-(4-phenoxyphenyl)amine 


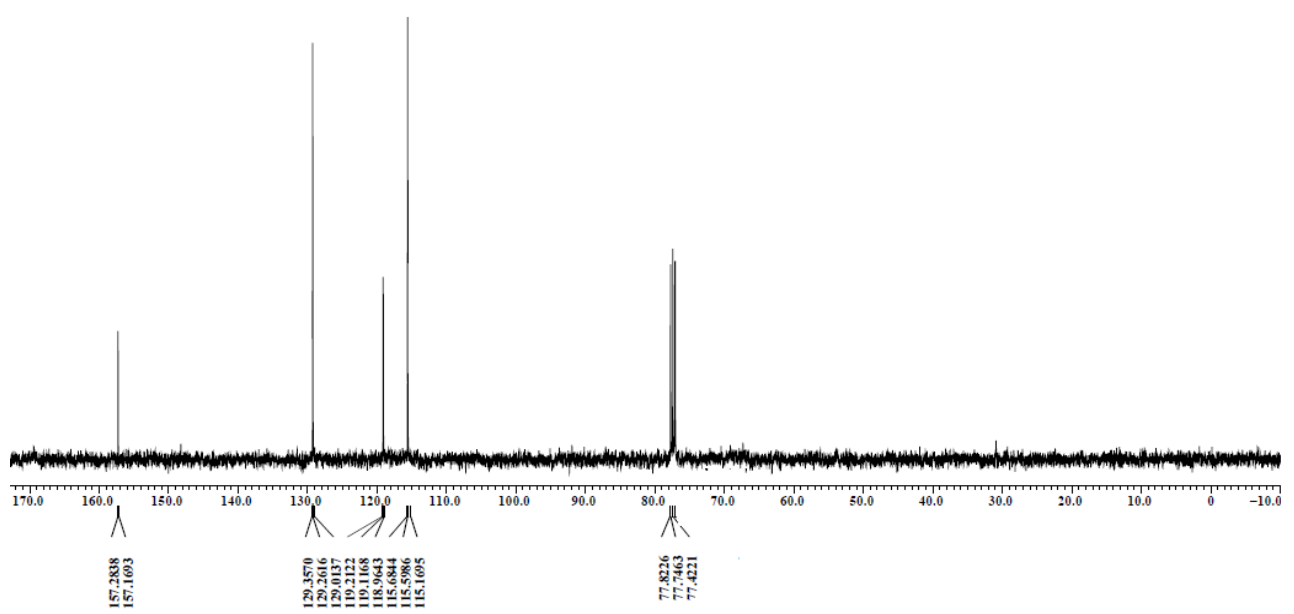

Figure 4. ${ }^{13} \mathrm{C}-\mathrm{NMR}$ for tris-(4-phenoxyphenyl)amine

${ }^{1} \mathrm{H}$ NMR (400 MHz $\mathrm{CDCl}_{3}, \delta$, ppm) for tris-(4-phenoxyphenyl)amine in Figure 3 shows the peak at $6.7135-6.7748(6 \mathrm{H})$ which corresponds to the protons of the triphenylamine, the peak at $6.8143-6.8345(12 \mathrm{H})$ corresponds to the proton of the aryl ring attached with the ethereal linkage, and the peak at 7.0866-7.1063(9 H) is for the peripheral protons of the aryl ring. Similarly ${ }^{13} \mathrm{C}$ NMR (400 $\mathrm{MHz}_{\mathrm{CDCl}}, \delta$, ppm) shown in Figure 4 represented all the carbon of the aryl ring at 157.28, 157.16, 129.35, 119.21 and 112.68.

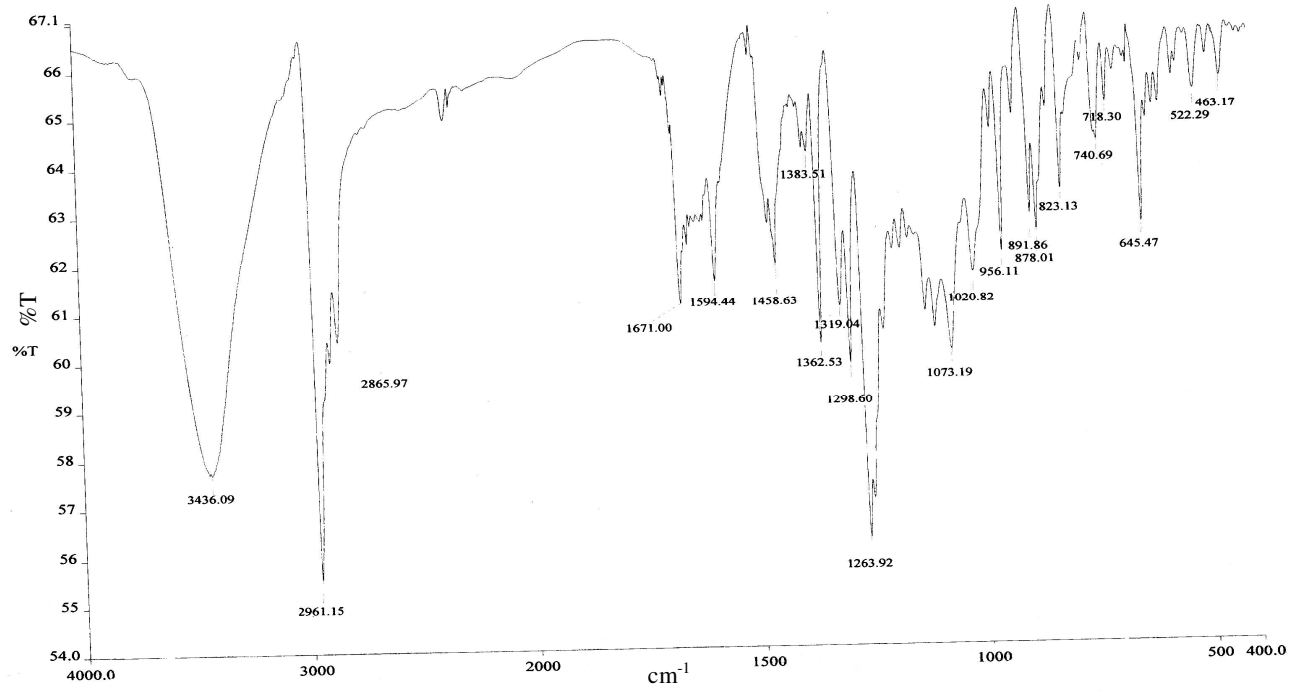

Figure 5. FT-IR spectra of tris-(4-phenoxyphenyl)amine

FT-IR spectra for the tris-(4-phenoxyphenyl)amine (Figure 5) was recorded in $\mathrm{KBr}$ as the medium. The stretching at $1020.82 \mathrm{~cm}-1$ corresponds for $-\mathrm{C}-\mathrm{N}$ bond and stretching peak at 1073.19 for the $-\mathrm{C}-\mathrm{O}$ ethereal linkage, the peak at $1362.53 \mathrm{~cm}-1$ is for $\mathrm{C}=\mathrm{C}$ of the aromatic ring and $2965 \mathrm{~cm}^{-1}$ for the $\mathrm{CH}$-of the aromatic ring. The broad peak at $3436.09 \mathrm{~cm}^{-1}$ was observed due to the presence of water in the medium of the spectra. 
The UV-Visible spectra for tris-(4-phenoxyphenyl)amine (Figure 6A) was recorded in tetrahydrofuran (THF) and shows a good maximum absorption $\left(\lambda_{\max } 308 \mathrm{~nm}\right)$ with an absorption of 1.408. The florescence for the tris-(4-phenoxyphenyl)amine (Figure 6B) was also recorded in tetrahydrofuran (THF) and gave a good emission $\left(\lambda_{\max } 410 \mathrm{~nm}\right)$ with intensity of 72.54 a.u.

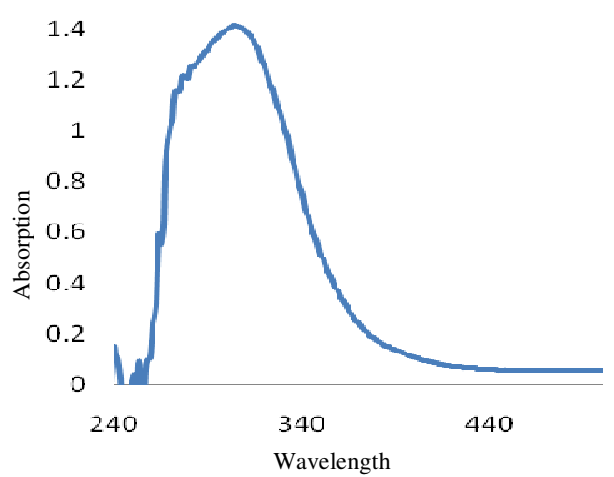

(A) UV-Vis spectra

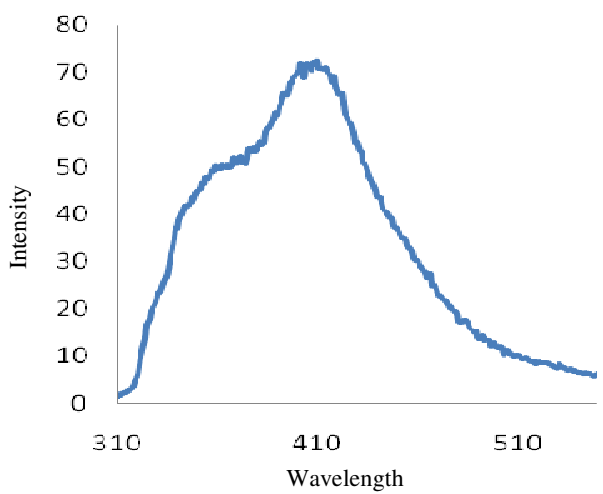

(B) Floroscence spectra

Figure 6. UV-Vis and floroscence spectra of tris-(4-phenoxyphenyl)amine

\section{Results and Discussion}

In this paper, the synthesis of triphenylamine based compounds with reaction between tris(4-bromophenyl)amine and phenolic compounds like phenol and naphthol is reported. The synthesized compounds were characterized and confirmed by ${ }^{1} \mathrm{H}$ NMR, ${ }^{13} \mathrm{C}$ NMR, FT-IR, UV-Vis. spectroscopy and florescence spectroscopy. In this paper tris-(4-phenoxyphenyl) amine, the tris-(4-bromophenyl)amine undergoes electrophillic substitution reaction with the nucleophile generated from the phenol by cleavage of the phenolic $-\mathrm{OH}$ bond. The study reveals that the lone pair of electrons in the ethereal linkage makes the energy increased and hence decrease in absorption towards lower wavelength. The synthesized compound and their derivatives will be used for biological and other applications.

\section{Acknowledgement}

The authors are thankful to the Head, USIC, Faculty of Science, University of Delhi, for providing IR and ${ }^{1} \mathrm{H}$ NMR spectra.

\section{References}

1. Gurnee E and Fernandez R, US Patent, 1965, 3172862.

2. Pope M, Kallman H and Magnante P, J Chem Phys., 1963, 38(8), 2042-2043; DOI:10.1063/1.1733929

3. Tang C W, US Patent, 1982, 4356429.

4. Tang C W and VanSlyke S A, Appl Phys Lett., 1987, 51(12), 913-915; DOI:10.1063/1.98799

5. Friend R, Burroughes J and Bradley D, WO Patent, 1990, 90, 13148.

6. Friend R and Burroughes J and Bradley D, US Patent, 1993, 5, 247190.

7. Cremer J and Bäuerle P, J Mater Chem., 2006, 16, 874-884; DOI:10.1039/B515657B 